\title{
Concept App Development for National Library of Virtual Manipulatives (NLVM): An Initiative
}

\author{
ABM Rezbaul Islam (Corresponding Author), Emma Bullock \\ Sam Houston State University, USA
}

Received: December 27, 2021 Accepted: February 7, 2022 Published: February 11, 2022

doi:10.5296/jet.v9i2.19384

URL: https://doi.org/10.5296/jet.v9i2.19384

\begin{abstract}
An NSF-supported project, the National Library of Virtual Manipulatives (NLVM), was started in 1999 to develop a library of entirely interactive and web-based virtual manipulatives or concept tutorials. It was mainly in Java applets for mathematics instruction (K-12 emphasis). The NLVM was a resource to develop mathematics classrooms from which teachers could independently draw upon. The materials were also significant in the mathematical training of both in-service and pre-service instructors. However, twenty years later, with the proliferation of touch-screen devices, this fantastic resource is in a crisis of becoming lost to future generations. It has been seen that many computers no longer run Java. Besides, the older version of Java used in the initial advancement of the NLVM is no longer supported. Hence, it is the right time to rethink the NLVM for current, widely available touch screen devices. This is essential to bolster the offerings for both prekindergarten, K- 12, and university settings. Keeping the situation in mind, we have constructed four virtual manipulative applications compatible with touch-screen devices based on the outdated java-script types of virtual manipulatives available through the NLVM. The four concept apps are being conducted with children in classroom settings starting in Spring 2021. It will allow for further revisions of the apps if necessary. Then, the apps from this project would be combined as part of a more significant, federally, or privately funded external grant proposal to re-imagine and broaden upon all the virtual manipulatives currently found in the NLVM.
\end{abstract}

Keywords: national library of virtual manipulatives, mathematics education, teaching and learning with technology, mobile app development, technology in education

\section{Introduction}

Mathematics is an essential part of any level of education. Learning mathematics requires student engagement and active learning (Scott Freeman, Sarah L Eddy, Miles McDonough, Michelle K Smith, Nnadozie Okoroafor, Hannah Jordt, and Mary Pat Wenderoth. 2014). Understanding their mistakes; active learning emphasizes student participation and adaptive learning. Student participation is essential for this reason, and dynamic mathematical objects 
focused on the touch screen can be an efficient approach (Moyer-PackenhaM, P. S., ShuMway, J. F., Bullock, E., Tucker, S. I., Anderson-Pence, K. L., Westenskow, A., \& Jordan, K. 2015). A research study was regulated by (Falloon, G. 2013), (Presmeg, N., Radford, L., Roth, W. M., \& Kadunz, G. 2016) that uses graphics, numbers, and words on the computer screen to connect the iconic (visual images, pictures) with the symbolic (words, numbers, symbols) mode. Thus (Moyer-Packenham, P. S., \& Bolyard, J. J. 2016) characterize virtual manipulatives as "an interactive, technology-enabled visual representation of a dynamic mathematical object" (p. 13). Technology will play a significant role in mathematics education in our school. The technological contribution is much more required when a global pandemic completely changes the traditional education system's dynamic. Face-to-face classes need to change to online classes, and for many reasons, that will be a new normal. We need to prepare for the online setting where learning is much more software-based. As the National Research Council suggested in 2020, High-quality, research-based digital tools can help teachers create conceptual and procedural knowledge. It will further help them add well-developed, included instructional.

The primary motivation of NLVM was to address the shortage of online tools for mathematical curriculum. The idea of NLVM is much more significant nowadays for the impact of COVID-19. However, for the advancement of technology, it needs to improve. The improvement will make it more feasible and easier to adopt in the classroom. The touch-screen devices make it more feature-rich, like- animation and slider control.

Nonetheless, from the financial perspective, virtual manipulatives are the right choice since they will save the stakeholders' costs by repaid many times. Once these virtual manipulatives are developed, everyone is related to in-class education. Teachers and students, regardless of the school district location, will have the same opportunities to use. Thus, the specific goals of this proposed project enclosed the re-imagined maturation of the following four NLVM applets into touch-screen compatible formats:

- Base Blocks: This applet was selected for diversified classroom applications from PK to university with pre-service elementary and secondary teachers. This applet can be helpful for pre-service teachers and children to value understanding and algebraic reasoning.

- Attribute Blocks: This applet was chosen for its significance in developing early one-to-one correspondence, spatial sense, and pattern recognition for early childhood settings.

- Measurement Conversion: This applet was chosen because of its wide application for both elementary and secondary settings as it scrutinizes the conversion of units for time, length, weight, area, and volume.

- Financial Math: This applet was selected because of its broad applications in upper elementary, secondary, and university settings in probing the concepts of principal, interest, length of loan repayment, and frequency of payments.

We developed four touch-screen compatible concept apps that we will then pilot in a future 
study. Our overall goal was to re-establish and improve the highly interactive learning tools in the NLVM that will appoint students in active learning in PK-16 settings. Our assumption includes checking out the tools' effectiveness in helping intended spectators to understand their relevant mathematical content.

The successful beta-testing and revision of these four concept apps will result in the possible availability of these apps as a free resource across the globe which will be most favorable to rural, underrepresented groups who want access to high-quality STEM teaching materials

\section{Related Works}

Recent research proposes essential design characteristics that increase conceptual understanding (Bullock, E. P., Roxburgh, A. L., Moyer-Packenham, P. S., Bektas, E., Webster, J. S., \& Bullock, K. A. 2021), (Moyer-Packenham, P. S., Lommatsch, C. W., Litster, K., Ashby, J., Bullock, E. K., Roxburgh, A. L \& Jordan, K. 2019) when using digital mathematics tools. The representation link between iconic and symbolic notations is vital since it includes bodily movements, visualizations, audio, and symbolic representations, following the mathematics content learning goals (MCLG) for app design features. These completed research projects work as an assisted guide in re-imagining the NLVM concept apps.

\section{Proposed Idea}

We are trying to revise the four concepts Apps and related instructional materials following a qualitative action research design (CITE). This research design is most efficient since it includes PK- 12 and universities' teaching environments. In both cases, the group size will be small. The researcher will collect video footage of users using those four apps. We plan to use the iPad as a part of the students' group activity. Those recording's primary goal is to find the user's movements using protocols proposed by (Moyer-PackenhaM, P. S., ShuMway, J. F., Bullock, E., Tucker, S. I., Anderson-Pence, K. L., Westenskow, A., \& Jordan, K. 2015). Also, there will be recorded interviews of those students about their experiences. Based on the recorded responses, we will make revisions as necessary. Nonetheless, the instructional materials will also undergo a four-round iterative advancement process.

\subsection{The Re-imagination of Four Concept Apps}

The first re-imagined applet will be of Base Blocks. It was selected because of its diversified classroom applications from prekindergarten to university. The current APP of NVLM is shown in Figure 1, and the re-imaged APP is given in Figure 2. 


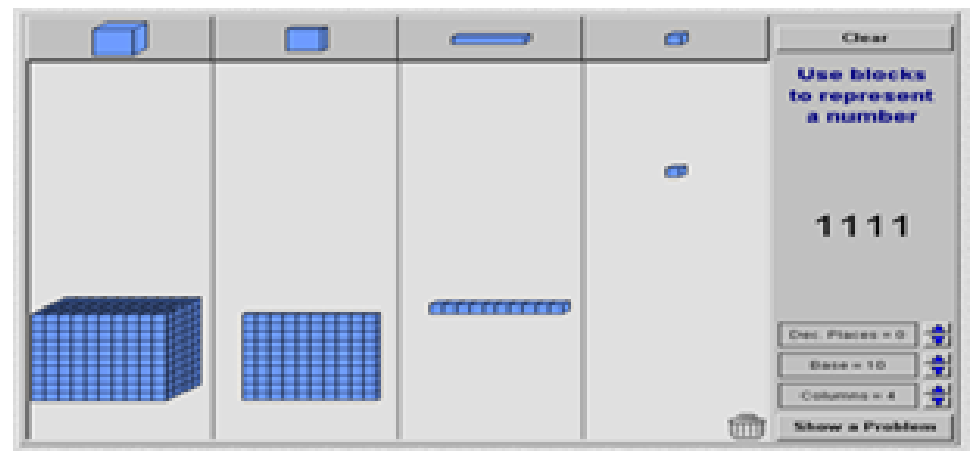

Figure 1. Current Base Block App at National Library of Virtual Manipulatives
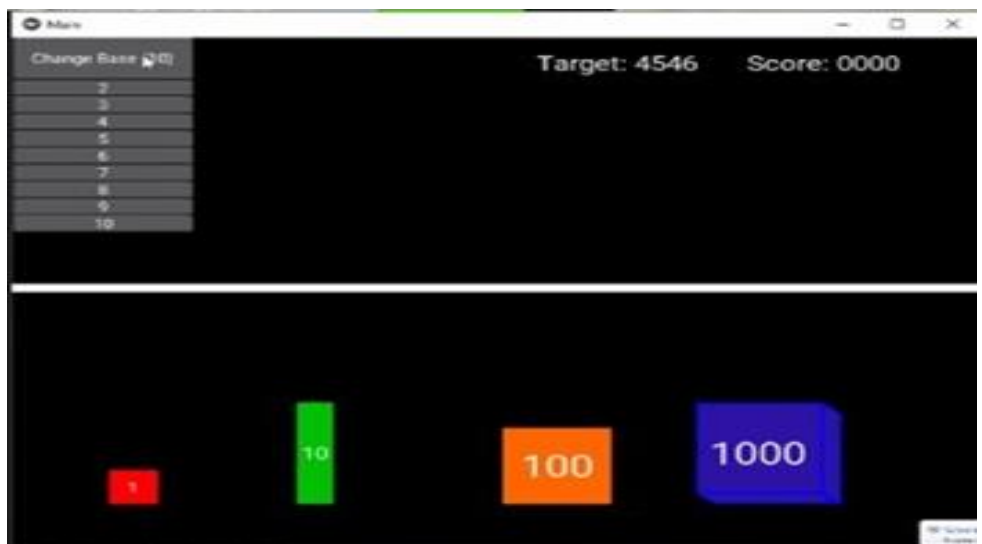

Figure 2. Re-imagined Base Block App

The re-imaged App provides simplicity and various volume shapes to understand the concept better. The use of different color codes also makes it easily distinguishable between positions. The second applet is attribute block. The current NVLM app is shown in Figure 3 and the re-imaged App in Figure 4.

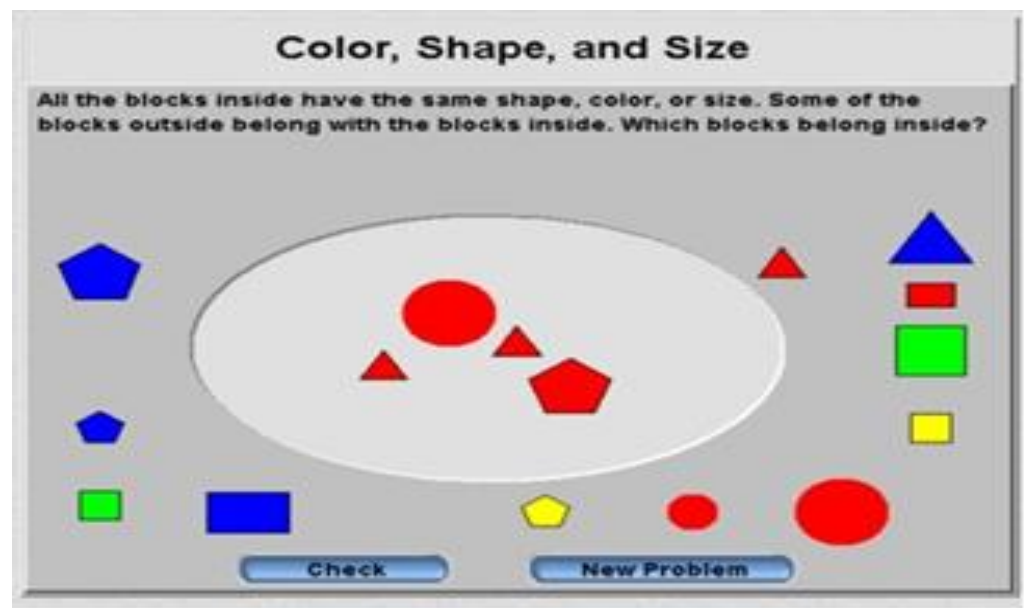

Figure 3. Current Attribute App at National Library of Virtual Manipulatives 


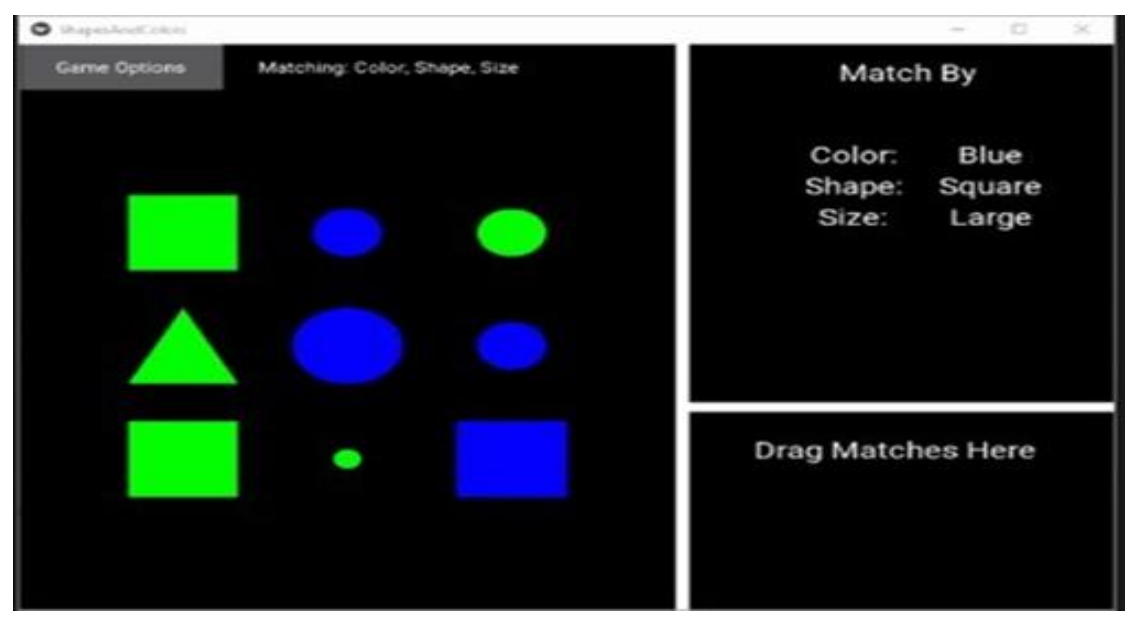

Figure 4. Re-imagined Attribute App

It is significant in developing early spatial sense and pattern recognition for early childhood. The re-imaged APP contains multiple instructions, and if the user is doing it correctly, a completion pop-up dialog box. The third re-imagined applet will be a Measurement Conversion tool that explores units' modification for time, length, weight, area, and volume. Thus, this tool has broad application for both elementary and secondary settings mostly.

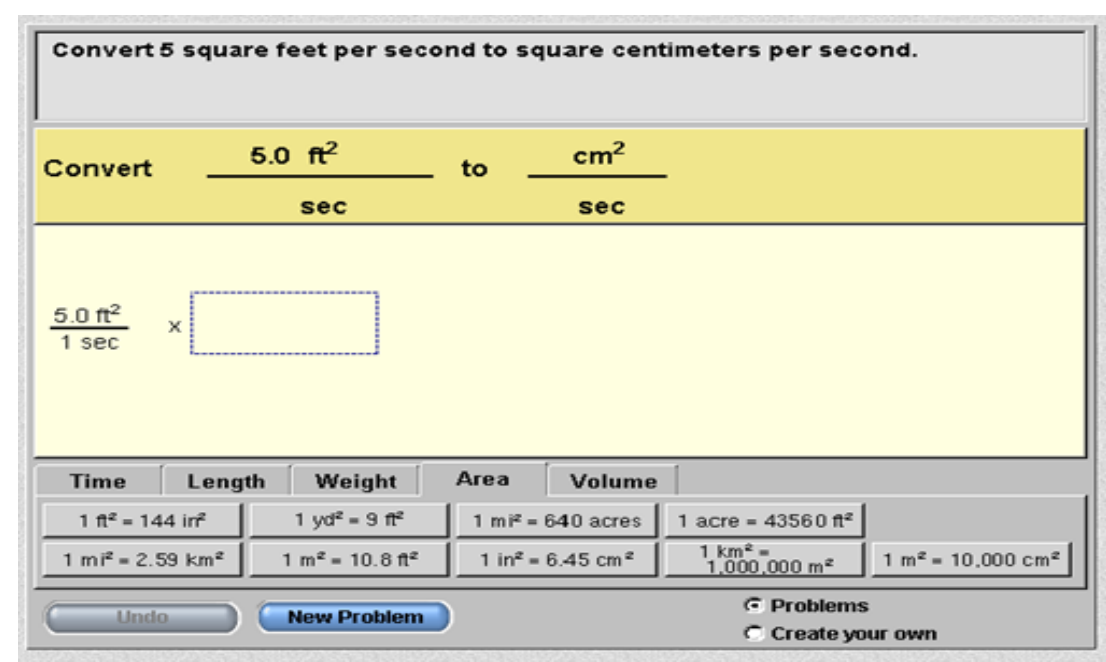

Figure 5. Current Measurement Block App at National Library of Virtual Manipulatives 


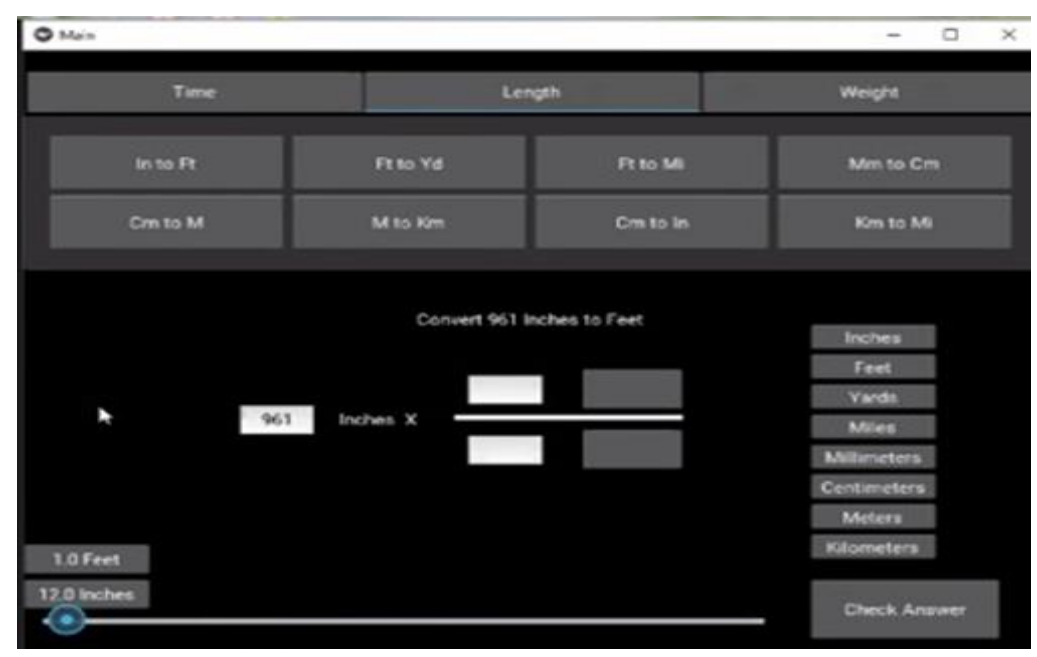

Figure 6. Re-imagined Measurement Block App

The current measurement app of NVLM is given in Figure 5 and the re-imagined version in Figure 6. The re-imaged version contains a slider at the bottom, which indicates the user's input. It is common to make mistakes for the measurements because of the fractional part. The slider will guide the error, and the user can correct that. This type of visualization is a crucial and essential part of learning. The fourth re-imagined applet will be a Financial Mathematics tool with broad applications in upper elementary, secondary, and university settings. It uses four sliders. The user can slide them for various values and see the respective changes. The current and re-imagined version is given in figure 7 and 8 , respectively.

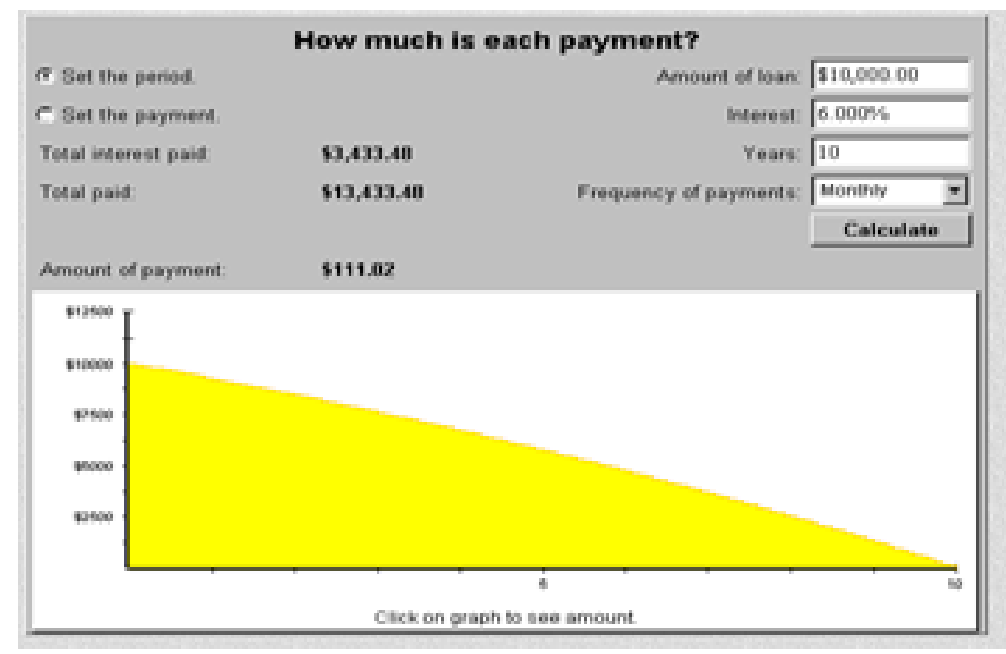

Figure 7. Current Financial Block App at National Library of Virtual Manipulatives 


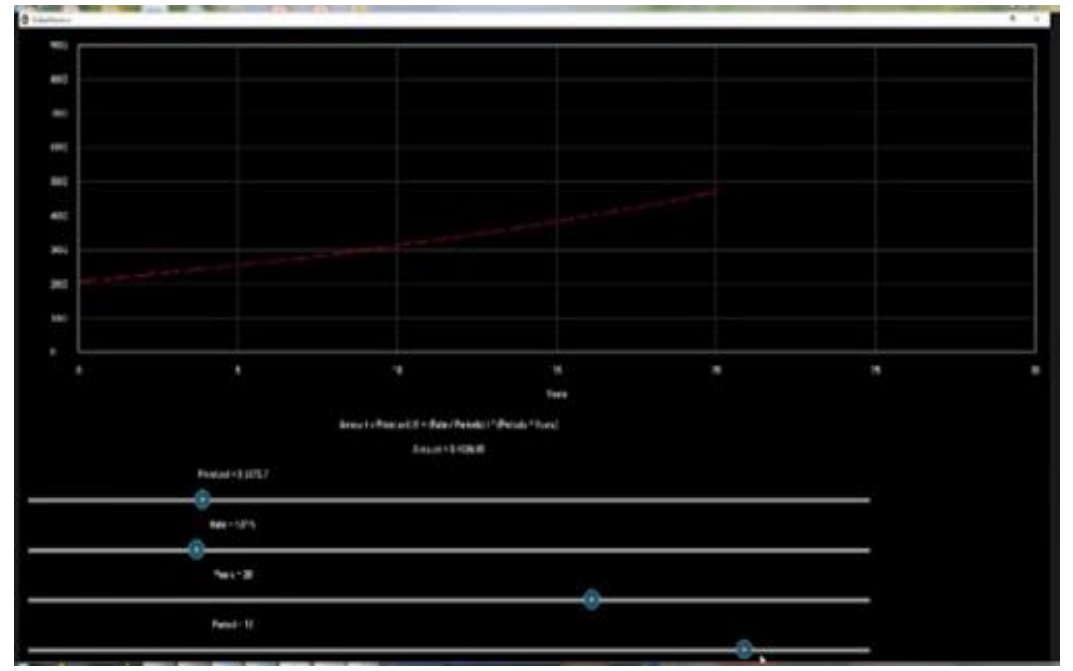

Figure 8. Re-imagined Financial Block App

\subsection{Developing the Apps}

In our work, we are trying to re-imagine the NVM concepts to cope with the current era of technology. Nowadays, most classrooms and students use touch-screen devices. If we can develop these apps adapted to those touch-screen devices, it will get much more attention and provide ease of use. We also keep in mind the varieties of iPod, iPhone, or any other tabs and their operating system software. These Touch-screen devices are usually run by two operating systems -iOS and Android. iOS is the operating system for Apple devices, whereas all other device uses the Android operating system. iOS and Androids are different in nature and independent in-app compatibility. However, some programming languages allow cross-platform compatibility for app development. We took advantage of this compatibility and used Kivy as a programming language to develop all the apps. Kivy is an open-source python library initially developed by MIT. This open-source library can run on various operating systems- Windows, Linux, iOS, Android, OS X, and Raspberry Pi.

One of the most prominent Kivy features is the same code can run in any operating system. It is very much flexible and easy to use. Besides, it can connect various input protocolstouch-screen, MacOS X trackpad, and Magic mouse. The Kivy is open source, so it is free of cost, and anyone can use it. There is no licensing for the Kivy software, which is different from the current technology used for NVLM. The current NVLM requires purchasing a license for the user, which is a bottleneck for many users. We want to develop apps available for all prospective users free of charge.

\section{Methodology}

\subsection{Study Design}

We will use a qualitative action research design to complete a rigorous beta-testing and revision of the four concept apps and associated instructional materials (Mertler, C. A. (Ed.). 2019). This research design is most appropriate because we will test the use of the concept apps in teaching environments in university and PK-12 classroom small group settings. 
Action research involves using an iterative reflective lens to methodologically work out steps to improve the user/learner experience. Thus, the data collected will evaluate the user/learner experience based on the work of (Bullock, E. P., \& Moyer-Packenham, P. S. 2019) and make appropriate revisions to the concept app code and relevant instructional materials through a four-round iterative process of improvement.

\subsection{Data Collection}

Using 6 GoPro cameras with chest mounts, we will collect video footage of the hand movements associated with PST $(\mathrm{N}=350)$ and PK-12 (N=45) (students using the concept apps on iPads as part of a small group instructional activity. (While we intend to have these tools available for Android compatible devices, as well, we chose iPads for this phase as they are the most widely used devices in educational settings.) Two rounds of data will be collected from PST and two rounds from PK-12 students. This data will allow us to observe the users' movements using the protocol adapted from (Bullock, E. P., \& Moyer-Packenham, P. S. 2019). In addition, brief audio-recorded semi-structured interviews will be conducted with each user to collect their experience with the apps right after the learning experience. Finally, brief audio-recorded semi-structured interviews will be shown with the small group teachers for their perspective of the user experience in the context of the learning activity.

\subsection{Data Analysis}

We will conduct a qualitative content analysis (Schreier, M. 2012) using two protocols developed by (Bullock, E. P., \& Moyer-Packenham, P. S. 2019) to code the GoPro camera footage for the quality of and user interactions with four types of design features within the concept apps: simultaneous linking (SL), creative variation (CV), focused constraint (FC), and guiding feedback (GF). In particular, we will analyze the videos for how these features were designed concerning the apps' mathematics content learning goal (MCLG) and how the users utilized these features. Then, we will cross-reference these findings with the interview data on the user/teacher's perceived helpfulness or hindering of the concept apps in the instructional activity. This analysis will then be used to create a list of revisions focused on improving the apps' SL, CV, FC, and GF features.

\section{Evaluation Criteria}

To evaluate the re-imagined App, we will follow the Coding Protocol for Assessing the Effectiveness of Mathematics Virtual Manipulatives (Adapted from (Bullock, E. P., Roxburgh, A. L., Moyer-Packenham, P. S., Bektas, E., Webster, J. S., \& Bullock, K. A. 2021), The details are given in table 1. 
Table 1. Evaluation Criteria

Mathematics-Content Specific Criteria

\begin{tabular}{lll} 
Coding & Types & Choice Response \\
protocol & & \\
\hline
\end{tabular}

Simultaneous Features act as linking mechanisms linking (SL) between different mathematical semiotic systems. In other words, they facilitate a connection between one mathematical representation and another representation in the game in more, or less abstract ways, either symbolically, visually, or audibly

Creative Features are elements in the game variation that support the development of the (CV) students' representations of mathematics.

Focused constraint (FC)
Features focus and constrain students to mathematical information that supports students' computation and abstraction.
Yes/No If yes, name at least one specific SL feature (i.e., movement of a slider is linked to increasing angle rotation of the rocket.

Yes/No If yes, name at least one specific $\mathrm{CV}$ feature (the game user can choose to represent a mathematical component in different ways individualized to the user-i.e., change colors to make things easier to see)

Yes/No If yes, name at least one specific FC feature (i.e., the user cannot go beyond a particular set of possible answers, thus forcing the user to stay in the ballpark of the correct concept)

Guiding

Features are representations that

Yes/No

If yes, name at least one specific GF feature (i.e., the game's visuals show the user how to find the correct answer-hints)

Semi-Structured Interview Protocol

What did you learn while you played/interacted with this App? 
- What did you notice about aspects of the App? (explore further any references to the

- $\quad \mathrm{SL}, \mathrm{CV}, \mathrm{FC}$, or GF design features) What helped you as you played/interacted with this

- App? What did not help you as you played/interacted with this App?

\section{Results}

By the end of the project, we expect to have four fully functioning, research-based concept apps based on the criteria of (Bullock, E. P., \& Moyer-Packenham, P. S. 2019) with accompanying instructional materials which can be used in a broad range of PK-16 scenarios. These will then be used to obtain funding to re-imagine the entire NLVM for touch-screen devices which will be freely available to anyone who wants to use them.

At the end of the research study, we will share and access the revised apps with participating PK-16 teachers, students, and their parents (if minors). To communicate these findings to digital math game developers and researchers, we will disseminate our findings through conference presentations at the Society for Information Technology and Teacher Education (SITE), AERA (Technology, Instruction, Cognition, and Learning Special Interest Group [TICL SIG]), Research Council of Mathematics Learning (RCML), and the American Society for Engineering Education (ASEE), among others. In addition, we will seek to publish these results in peer-reviewed journals such as the Journal of Computer-Assisted Learning, Computers in Education, Technology, Knowledge and Learning, and Contemporary Issues in Technology and Teacher Education (CITE), among others.

It is possible that COVID19 could play a role in delaying our activities if vaccine efforts have not been expansive enough by the proposed start date of the project. Also, recruiting underrepresented minority and women research assistants is sometimes challenging. This is always difficult in rural areas. Finally, it will be essential to coordinate care with local schools when PSTs teach their lessons using the concept apps. Besides, Go-Pro Cameras are limited in capturing the image right in front of the camera. While we anticipate this accurately capturing the needed movement data, we will need to carefully cross-reference this with the interview and field notes data to ensure we have the correct revision understandings.

\section{References}

Bullock, E. P., \& Moyer-Packenham, P. S. (2019). The Importance of Shared Vision and Stakeholder Influence on K-12 School Leaders' Efforts to Improve Student Mathematics Achievement. Stakeholder Engagement: Improving Education through Multi-Level Community Relations, 33.

Bullock, E. P., Roxburgh, A. L., Moyer-Packenham, P. S., Bektas, E., Webster, J. S., \& Bullock, K. A. (2021). Connecting the dots: Understanding the interrelated impacts of type, quality, and children's awareness of design features and the mathematics content learning goals in digital math games and related learning outcomes. Journal of Computer Assisted Learning, 37(2), 557-586. https://doi.org/10.1111/jcal.12508

Falloon, G. (2013). Young students using iPads: App design and content influence their learning pathways. Computers \& Education, 68, 505-521. 
https://doi.org/10.1016/j.compedu.2013.06.006

Mertler, C. A. (Ed.). (2019). The Wiley handbook of action research in education. John Wiley \& Sons. https://doi.org/10.1002/9781119399490

Moyer-Packenham, P. S., \& Bolyard, J. J. (2016). Revisiting the definition of a virtual manipulative. In International perspectives on teaching and learning mathematics with virtual manipulatives (pp. 3-23). Springer, Cham. https://doi.org/10.1007/978-3-319-32718-1_1

Moyer-Packenham, P. S., Lommatsch, C. W., Litster, K., Ashby, J., Bullock, E. K., Roxburgh, A. L., ... \& Jordan, K. (2019). How design features in digital math games support learning and mathematics connections. Computers in Human Behavior, 91, 316-332.

https://doi.org/10.1016/j.chb.2018.09.036

Moyer-PackenhaM, P. S., ShuMway, J. F., Bullock, E., Tucker, S. I., Anderson-Pence, K. L., Westenskow, A., ... \& Jordan, K. (2015). Young children's learning performance and efficiency when using virtual manipulative mathematics iPad apps. Journal of Computers in Mathematics and Science Teaching, 34(1), 41-69.

Presmeg, N., Radford, L., Roth, W. M., \& Kadunz, G. (2016). Semiotics in mathematics education (p. 40). Springer Nature. https://doi.org/10.1007/978-3-319-31370-2

Schreier, M. (2012). Qualitative content analysis in practice. Sage publications.

Scott, F., Sarah, L. E., Miles, McDonough, Michelle, K. S., Nnadozie, O., Hannah, J., \&Mary, P. W. (2014). Active learning increases student performance in science, engineering, and mathematics. Proceedings of the National Academy of Sciences, 111(23), 8410-8415. https://doi.org/10.1073/pnas.1319030111

\section{Copyright Disclaimer}

Copyright for this article is retained by the author(s), with first publication rights granted to the journal.

This is an open-access article distributed under the terms and conditions of the Creative Commons Attribution license (http://creativecommons.org/licenses/by/4.0/). 